\title{
Cognizance and intervention of a pharmacist in antimicrobial stewardship program among students of health care professionals, a cross sectional study
}

\begin{abstract}
Antimicrobial stewardship is a program which promotes the accurate use of antibiotic in which, a team is formed and under this team the strategies are made to overcome the misuse and inappropriate practice of antibiotics which leads to antibiotic resistance eventually. In our study we make a validated structured questionnaire which contained 12 close ended questions regarding this program and pharmacist intervention in antibiotic stewardship and was filled by the students of health care professionals, so far the study was done by the time span of 6 months from January 2016 till July 2016.Total 109 forms was filled by diverse eminent health care members of different institute, out of 109 only 29 personnel's knew this term. Hence it is obvious that the neglecting circumstances of our students towards their profession.
\end{abstract}

Keywords: antimicrobial stewardship, antibiotic, resistance, awareness, health care professionals
Volume 3 Issue I - 2017

Syeda Sarah Abbas, ${ }^{1,2}$ Syed Yousuf Ejaz, ${ }^{2}$ Safila Naveed,' Fatima Qamar,' Samina Alam,' ZohraBarkat Ali,' Sania Zehra,' Sehrish Kirn' 'Faculty of Pharmacy, Jinnah University for Women, Pakistan ${ }^{2}$ Department of Pharmaceutics, University of Karachi, Pakistan

Correspondence: Syeda Sarah Abbas, Lecturer at Faculty of Pharmacy, Jinnah University for Women, Pakistan, Email syedasarahabbas@yahoo.com

Received: August 27, 2016 | Published: January 31, 2016

\section{Introduction}

Antibiotics are the valuable medicines which were made to kill or to stop the growth of bacteria. Fleming Alexander was the first person to discover first antibiotic named penicillin which is used to stop the growth of bacteria. ${ }^{1}$ Bacteria's are classified under gram positive, gram negative and anaerobic. But nowadays bacteria's are little more efficient and many antibiotics are resistant to it. ${ }^{2}$ Resistance of antibiotics or antibiotic resistance means failure of antibiotic against any infections or the ability of bacteria to resist the effects of antibiotic. The main mechanism to resist the use of antibiotic is the DNA of bacteria change mutates and altered. ${ }^{3}$ There is many causes of failure of antibiotics or resistance of antibiotics.

The main causes of resistance is misuse, overuse, under/inadequate use of antimicrobials. It may include

A. Antibiotics are prescribed unnecessarily.

B. Antibiotic administration is delayed in critically ill patients.

C. Broad-spectrum antibiotics are used too generously, or when narrow-spectrum antibiotics are used incorrectly.

D. The dose of antibiotics is lower or higher than appropriate for the specific patient.

E. The duration of antibiotic treatment is too short or too long.

F. Antibiotics available as over the counter.

For this reason many hospitals introduce a programme named antimicrobial stewardship. The word antimicrobial stewardship refers to the rational use of antibiotic with right drug, dose, and routeof administration and duration of antibiotic. ${ }^{4,5}$ The goal of antimicrobial stewardship is to minimize the consequences of antimicrobial uses refer to toxicity and emergence of resistanceor to increase pharmacoeconomic. ${ }^{6}$

\section{Antimicrobial stewardship team}

To overcome resistance and to control infection a team is formed to work.
A. Clinical pharmacist
B. Clinical microbiologist
C. Physician
D. Administrator

\section{Strategy to achieve goal}

Antimicrobial restriction is the method it is either through formulary limitation by this method or by the requirement of preauthorization and justification-is the most effective method of achieving the process goal of controlling antimicrobial use.

\section{Role of pharmacist}

The role of pharmacist is to eradicate infection and to control infection by educating healthcare professionals, to promote les or optimum use of antimicrobial use and to reduce transmission.

\section{Methodology}

We designed a validated structured questionnaire of closed ended questions. This is cross sectional study conducted in Karachi, Pakistan of sample size 120 which illustrate the awareness of antimicrobial stewardship among health care professionals including nurses, doctors, pharmacist, physiotherapist and pathologist. An informed consent form was filled by the authorities' body of diverse health care institutes; quantitavely total 109 forms were filled back by different health care professionals. The participants were selected randomly including students of different universities. The data was then analyzed statistically by spss $\mathrm{v} 20$. 


\section{Observations}

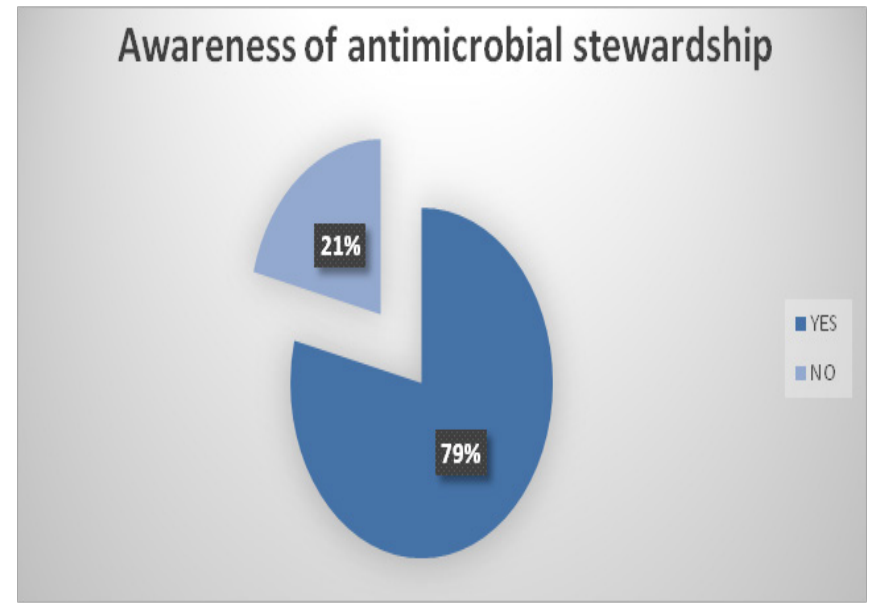

Figure I Awareness about antimicrobial stewardship

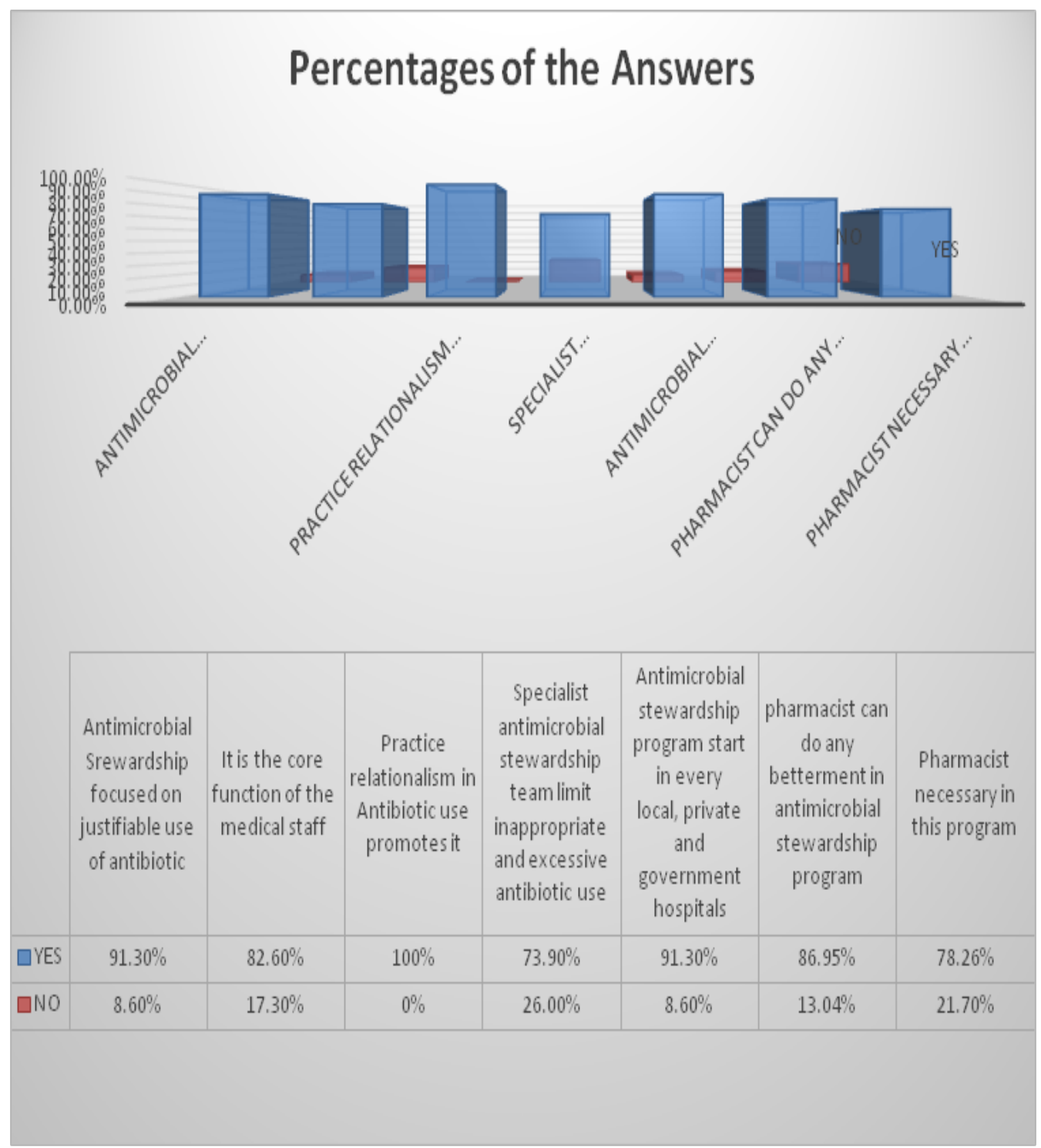

Figure 2 Complete analysis of responses. 


\section{Result}

From above study we concluded that out of 100 , only $21 \%$ health care professional knows and aware about the terminology "antimicrobial stewardship" and $79 \%$ health care professional did not knew about this program. $21 \%$ health care professional who knew that ask to fill further questions in which $91 \%$ health care professional said that this program focused on accurate and justifiable use of antibiotic. $82 \%$ health care professional said that it is a core function of medical staff, $100 \%$ health care professional this program promotes rationalism of antibiotic use, $73 \%$ health care professional said that Specialist antimicrobial stewardship team limit inappropriate and excessive antibiotic use, $91 \%$ health care professional said that antimicrobial stewardship program start in every local, private and government hospitals, $86 \%$ said that pharmacist can do any betterment in this program and $78 \%$ health care professional said that pharmacist are necessary.

\section{Discussion and conclusion}

The reason behind this study was to evaluate the level of awareness and exposure of students towards different health care programs which are running side by side to improve health care and rationale use of drugs. Many health care professionals did not know about antimicrobial stewardship. Few of them who knew this term also gave wrong answers like $8 \%$ said that antimicrobial stewardship did not focused on justifiable use of antibiotics and even $21 \%$ health care professionals did not know that pharmacist is necessary for this program so in a nut shell we concluded that our health care professionals lack awareness regarding this foremost program which contribute a major part in healthcare.

\section{Acknowledgements}

None.

\section{Conflict of interest}

The author declares no conflict of interest.

\section{References}

1. Fleming A. The Penicillin Finder Assays its Future. USA: The New York Times; $1945.21 \mathrm{p}$

2. Boucher, HW, Talbot GH, et al. Bad buds, no drugs: no ESKAPE! An update from the Infectious Diseases Society of America. Clin Infect Dis. 2009;48(1):1-12.

3. Shlaes DM, Gerding DN, John JF, et al. Society for Healthcare Epidemiology of America and Infectious Diseases Society of America Joint Committee on the Prevention of Antimicrobial Resistance: guidelines for the prevention of antimicrobial resistance in hospitals. Infect Control Hosp Epidemiol. 1997;18(4):275-291.

4. Pestonik SL, Classen DC, Evans RS, et al. Implementing antibiotic practice guidelines through computer assisted decision support: clinical and financial outcomes. Ann Intern Med. 1996;124(10):884-890.

5. Owens RC, Fraser GL, Stogsdill P. Antimicrobial stewardship programs as a means to optimize antimicrobial use. Insights from the Society of Infectious Diseases Pharmacists. Pharmacotherapy. 2004;24(7):896-908.

6. Dellit TH, Owens RC, McGowan JE, et al. Infectious Diseases Society of America and the Society for Healthcare Epidemiology of America guidelines for developing an institutional program to enhance antimicrobial stewardship. Clin Infect Dis. 2007;44(2):159-177.

7. Pharmacists. ASHP guidelines on the pharmacist's role in the development, implementation and assessment of critical pathways. Am J Health-Syst Pharm. 2004;6(9):939-945. 